\title{
GFAP AND NF EXPRESSION IN BRAIN TISSUE IN CHILDREN AND ADULTS AFTER FATAL TRAUMATIC BRAIN INJURY
}

\author{
A. Barzdina ${ }^{1}$, M. Pilmane ${ }^{2}$, A. Petersons ${ }^{3}$ \\ ${ }^{1}$ Department of Paediatric Intensive Care, Riga Stradin's University \\ ${ }^{2}$ Institute of Anatomy and Anthropology, Riga Stradins University \\ ${ }^{3}$ Department of Paediatric Surgery, Riga Stradins University
}

\begin{abstract}
Background and objectives. Still, there is almost no information about the role of biomarkers in the pathological processes of the brain in those patients, which die immediately after the injury, and those, which die several hours after the trauma. Design and Settings. A retrospective study. The human brain tissue material from the archive of the Institute of Anatomy and Anthropology in Riga Stradins University (RSU). Methods. We used the brain tissue material from the trauma and counterstroke spots of 28 patients. Brain tissue specimens were routinely fixed, embedded into paraffin, cut in $5 \mu \mathrm{m}$ thick slides. For immunohistochemistry we used monoclonal antibodies against NF proteins to detect axonal injury and monoclonal antibodies against GFAP to detect astrocytes. Results. Statistical correlation was seen between the lethal cases and survived in the brain tissue in the areas of counterstroke between lethal cases and survived for NF and GFAP presence $(p=0.017)$ The data was compared, by dividing patients into groups of children and adults. Each of these groups was divided into 2 sub-groups. Statistically significant differences were noted between the lethal and the survived cases in the group of children for GFAP (Mann-Whitney U Test, $p=$ 0.015 ) and in the group of adults for NF in the area of the counterstroke (Mann-Whitney U Test, $p=0.019$ ). Conclusions. Higher quantities of intermediated filaments such as GFAP and NF are characteristic in the patients who survived after a head trauma in comparison to those, who died on the spot of the accident. Children under 2 years of age with severe head trauma have more dynamic glial cell reaction than other patients.
\end{abstract}


Key words: Severe traumatic brain injury; secondary brain injury; structural brain damage; reactive astrocytes; glial fibrillary acidic protein; neurofilament; astrogliosis.

\section{INTRODUCTION}

The role of biomarkers in prognosticating therapy and the result of severe traumatic brain injury (TBI) have increased in the past decades [1]. The traumatic brain injury is a common cause of death and disability in children and adults of all ages. After the primary injury, the secondary injury to the brain follows. This process causes the multiple morphological damage of the brain tissue releasing a lot of substances, which play an important role in the recovery processes and in the outcome in general. Severe head trauma studies of the past couple of years are dedicated to exploring biomarkers and their role in the pathological processes that take place right after the moment of trauma; special attention is focused on those biomarkers that characterize the structural brain injury, thus determining neuronal, axonal and glial damage, and the regeneration potential. There are two main courses in these studies: 1. The biomarkers that characterize brain tissue damage, determining neuronal, axonal and glial damage, and the regeneration potential. 2. The mediators of the secondary brain damage are studied [11]. When the structural integrity of the nervous system is compromised, glial cells are activated to restore homeostasis [17]. Astrocytes are the multifunctional cells that play an essential role in homeostasis, and contribute to the information processing in physiological processes; they are also capable to generate a response to any kind of insult to the central nervous system (CNS). Within a few hours after virtually any type of brain injury, the surviving astrocytes in the affected region exhibit hypertrophy, and they proliferate in a process that is called reactive astrogliosis $[5 ; 19]$. Reactive astrocytes increase the expression of their structural proteins, glial fibrillary acidic protein (GFAP) [5] and neurofilament (NF) from intermediate filaments (IF) group. Immediately after the injury reactive astrocytes interweave their processes to form a glial scar that can impede axon regeneration. Most research is focused on the direct protection of neuronal cells; however, non-neuronal cells, like astrocytes can exert an active role in the pathogenesis of TBI [12]. Now the evidence has increased, and the modalities and dynamics of the 
astrocytal response to damage are crucial to the outcome of brain pathology and the degree of neurological damage. Taking this into account, astrogliosis appears to be an appealing therapeutic target for the implementation of endogenous repair in the CNS [22].

There is almost no information about the role of biomarkers in the pathological processes of the brain in those patients, who die immediately after the injury, and those, who die several hours after the trauma. The aim of our work was to detect differences of brain tissue reactions in two different spots of the human brain, after traumatic brain injury in pediatric patients and in adults who died immediately after the trauma and those, who were treated for several hours after the accident.

\section{MATERIALS AND METHODS}

We used the material from the archive of the Institute of Anatomy and Anthropology in Riga Stradins University (RSU) brain tissue from the trauma and counterstroke spots of 28 patients (permission of the RSU Committee of Ethics Nr. E-9(2) - 17.12.2009.). Patients were divided into 2 groups. All patients had a severe head injury (SHI). The patients with multiple traumas were excluded. In the beginning all 28 patients were divided into 2 groups: 20 patients, who died in the place of the accident ( 7 children and 13 adults), and 8 patients, who survived and received therapy for different amounts of time ( 5 children and 3 adults). Then all 28 patients were divided into the following 2 groups: group 1 included children up to 18 years old: 7 children died on the spot of the accident $(1,4,10,11$ years old, 2 children were 15 and 2 were 17 years old), and 5 children, who were hospitalized and received treatment for more than 24 hours (a 2-year-old, hospitalized for 48 hours, a 16-yearold, hospitalized for 78 hours, a 17-year-old, hospitalized for 36 hours and another 17-year-old, hospitalized for 168 hours).

Group 2 consisted of 16 adults, 18 to 61 years old, 13 of them died on the spot of the accident, but 3 were hospitalized after SHI and received therapy for more than 24 hours (a 36 year old, hospitalized for 15 days (360 hours), a 37-year-old, hospitalized for 11 days (264 hours), and a 40-year-old, hospitalized for 7 days (168 hours). In all the groups we analyzed GFAP and NF in the areas of impact and counterstroke.

Brain tissue specimens were fixed in $10 \%$ formaldehyde. Then tissue specimens were embedded into paraffin, cut in $5 \mu \mathrm{m}$ thick slides. For 
immunohistochemistry we used monoclonal antibodies against NF proteins to detect axonal injury (Monoclonal Mouse Anti - Human Neurofilament Protein Clone 2F11, working dilution 1:100, Dako, Denmark) and monoclonal antibodies against GFAP to detect astrocytes injury (Monoclonal Anti - Human Glial Fibrillary Acidic Protein Clone 6F2, working dilution 1:100, DakoCytomation, Denmark).

Sections were de-parafinised in xylene, and kept in absolute ethanol, then rinsed with PBS pH 7,4 (10 min), put into 4\% citrate buffer solution, and placed in the microwave for $20 \mathrm{~min}(750 \mathrm{~W})$. After cooling and rinsing with PBS the tissue samples were covered in $150 \mu 13 \%$ hydrogen peroxide (10 min.). After rinsing with PBS the primary antibody $(30 \mu \mathrm{l})$ was applied for 2 hours, then LSAB + LINK (linked streptavidin antibody) was applied for $30 \mathrm{~min}$, LSAB + KIT (streptavidin connected with enzyme peroxidase) was applied for 25 min, and DAB color reaction for 10 min. Finally, routine hematoxyline and eosine staining was performed in each sample (05B1003 Eosin Y Alcoholic Solution and 05M06002 Mayers Hematoxylin, Bio optica, Italy).

The semi - quantitative method was used for the quantification of NF- and GFAP-containing cells in the white substance of brain - on the spot of the trauma and on the spot of the counterstroke. The designations were as follows: 0 - negative reaction; $0 /+-$ occasional positive structures in the field of view; +- few positive structures in the field of view; ++- moderate number of positive structures in the field of view; +++- numerous positive structures in the field of view; ++++abundance of positive structures in the field of view [20].

Further data processing was done with SPSS (Statistical package for social sciences for Windows 17.0 ASV) software, using the nonparametrical statistical method, group and correlation methods.

\section{RESULTS}

In the overview slides the tissue in the area of the impact was relatively less damaged than in the area of the counterstroke. Plethoric blood vessels and glial proliferation was noted in the surviving patients. In all the groups glial edema was seen.

From 20 patients who died in the place of the accident NF in the area of the direct impact was detected only in 3 children and no adults. From 
8 patients who survived the trauma, in 7 we noticed NF in the area of the impact. NF in the area of the counterstroke was observed to be significantly higher in the survived patients compared with the patients who died in the place of the accident. When calculating the Spearmen correlation, a statistical correlation was seen in the NF presence in the brain tissue in the areas of the counterstroke between lethal cases and the survived $(\mathrm{p}=0.017)$. There was no statistically significant correlation in the NF presence on the spot of trauma between the survived and the lethal cases. The correlation coefficient " $r$ " is low because out of 28 patients only 10 had a positive NF reaction in the area of trauma.

The data about GFAP is similar. We detected the presence of GFAP on the spot of trauma only in 2 children, who died in the place of accident, and in 7 survived patients ( 5 children and 2 adults). The quantity of GFAP in the area of the counterstroke was noted higher in all 28 patients, both lethal cases and survived groups. When calculating the Spearmen correlation, a statistically significant correlation was seen in GFAP presence in the brain tissue in the areas of the counterstroke between survived and lethal cases $(p=0.017)$. There was no statistically significant correlation in GFAP presence on the spot of the trauma between the survived and lethal cases; from 28 patients only in 9 positive GFAP reaction was detected.

The data were compared, by dividing patients into groups of children (see Figure 1) and adults (see Figure 2), and each of these groups were divided into 2 sub-groups. The Mann-Whitney U Test and the Wilcoxon Signed Ranks Test were performed. Statistically significant differences were noted in the group of children, between lethal and survived cases in GFAP in the area of the counterstroke (Mann-Whitney U Test, $\mathrm{p}=0.015$ and Wilcoxon Signed Ranks Test, $\mathrm{p}=0.030)$. Statistically significant differences were noted in the group of adults, between lethal and survived cases in NF in the area of the counterstroke (MannWhitney U Test, $\mathrm{p}=0.019$ and Wilcoxon Signed Ranks Test, $\mathrm{p}=0.025)$. 


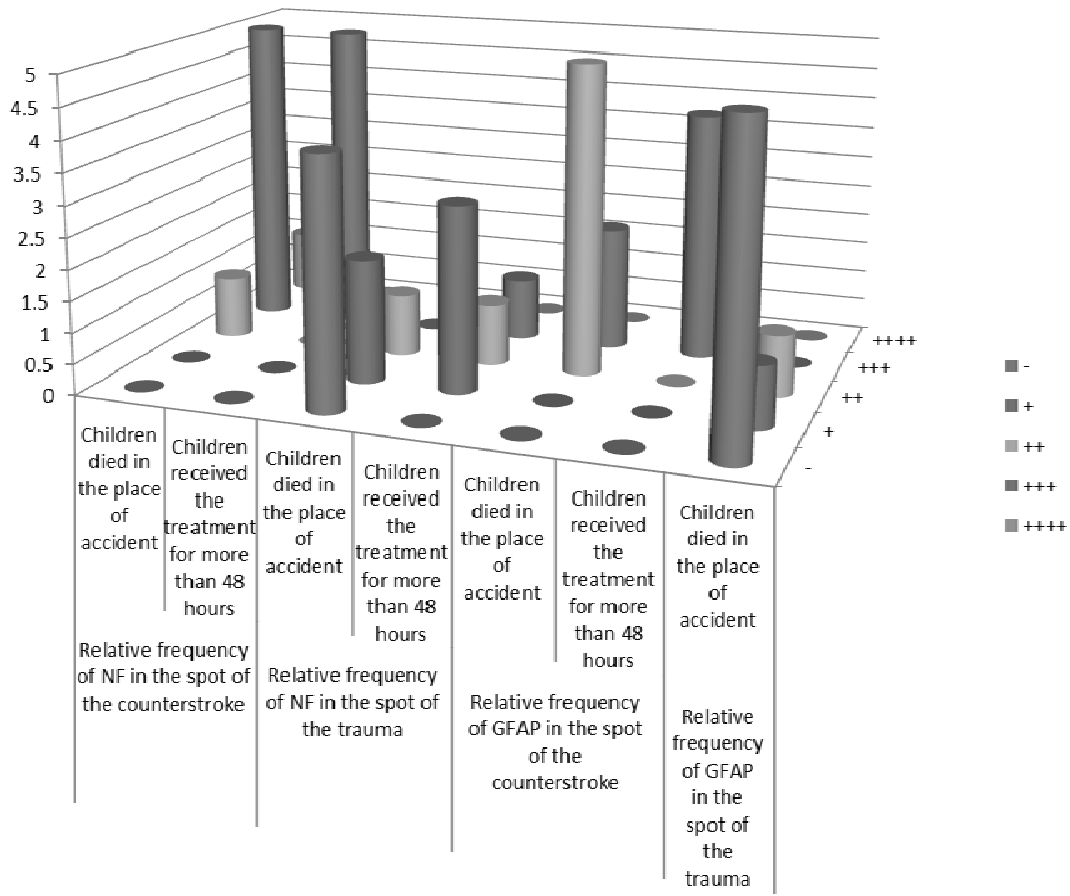

$\mathrm{NF}$ - neurofilament, GFAP - glial fibrillary acidic protein

Relative number abrevations:

-lack of cellss containing NF and GFAP structures

+ few positive structures

++ moderate number of positive structures

+++ numerous positive structures

++++ abundance of positive structures

Figure 1. Relative frequency of intermediate filaments GFAP and NF in the children who died in the place of the accident and who received the treatment for more than 48 hours. 

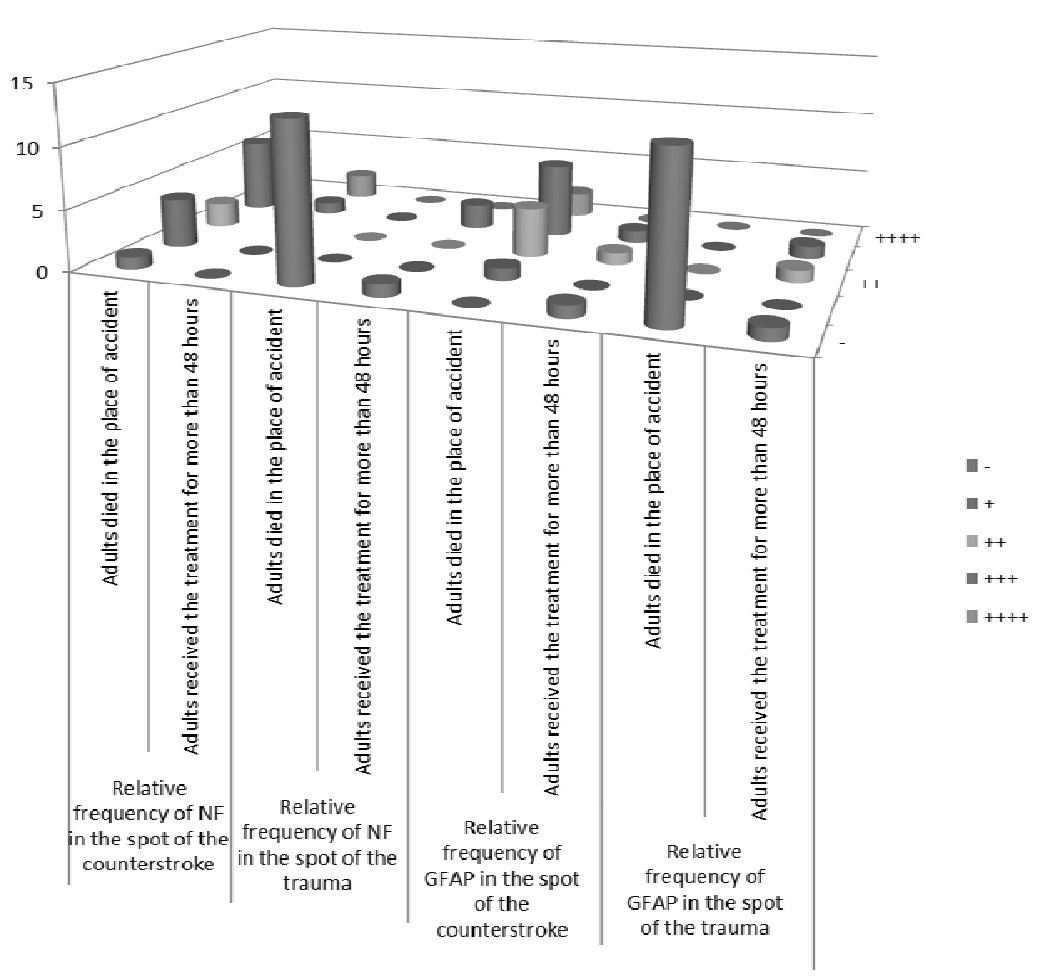

NF - neurofilament, GFAP - glial fibrillary acidic protein

Relative number abrevations:

++ moderate number of positive structures

- lack of cellss containing NF and GFAP structures

+++ numerous positive structures

+ few positive structures ++++ abundance of positive structures

Figure 2. Relative frequency of intermediate filaments GFAP and NF in the adults who died in the place of accident and who received the treatment for more than 48 hours.

\section{DISCUSSION}

The primary brain damage occurs at the same time as the traumatic impact; within a few minutes, hours and days the secondary damage occurs, which is characterized macroscopically as posttraumatic 
bleeding, and forming of a secondary contusion focus and the hypoxic zone of penumbre [7]. Microscopically it is characterized as a cascade of biochemical reactions, in which the damaged blood brain barrier (BBB) is actively enrolled, and it is acting as the neuro-inflammatory mediator producer [6]. Release of pro-inflammatory molecules can potentially induce further stress to compromised penumbral regions of the injured brain [23]. Aside of these processes the brain injury induces the activation of the intermediated filament (IF) structures. Glial cells participate in the inflammatory response. In particular, microglial cells are strongly activated following the injury and play an important role in the phagocytosis of the injured brain tissue [24]. Astrocytes are also activated in glial fibrillary acid protein (GFAP) expression. Astrocytes participate in the immune response of brain and form a permanent glial scar that physically and biochemically inhibits axonal regeneration and re-myelination. Reactive gliosis has been thought to be the major impediment to axonal re-growth after an injury, the formation of the glial barrier around a lesion site is also an advantage, because it protects the intact CNS tissue from secondary lesions [2]. In those first minutes of acute traumatic brain injury prominent reactive astrocytosis is seen. Astrocyte responses to injury are aimed at both protecting the nervous system, and sealing off damaged area, leaving the heavily injured zone to its natural degeneration, while preserving the less affected tissue. They may lead to reparative or destructive outcomes depending on the context in which they occur, for example, the extent and the type of injury, and the time point after damage [4].

In this study we examined the reaction of the white substance in the spot of the impact and the counterstroke by detecting the presence of two intermediated filaments in the structures of the brain tissue. IF in the human nervous system can be found both, in astrocytes and neurons, thus in the case of the fatal brain injury the information about the reaction of astrocytes was given by GFAP, and the presence of NF showed the damage of neurons [14].

Our result shows that in both, patients died on the spot of the injury and those who survived the injury moment NF and GFAP in the white substance of the brain was found less in the spot of the impact than in the spot of the counterstroke. In most patients, who died on the spot of the injury no NF and GFAP were found. Most of the studies concluded so far were made with animals, mostly rats, determining how quickly 
after the injury astrocytal and microglial reaction appears, and measuring the presence of neurinflammatory cytokines. In rats the immune-reactive activation of microglia in peri-lesional regions can be seen only after 24 hours after the injury [25]. In our study in children younger than 2 years, who died on the spot of the injury we found the presence of NF and GFAP on the spot of the counterstroke.

Analyzing the data among groups, children versus adults, in children more pronounced amounts of NF and GFAP were seen in both groups, who died in the place of accident, and in those who were treated for more than 48 hours after the injury. We did not note the presence of NF and GFAP in any of the adults, who died in the place of the inury, but in some children, who died in the place of the accident, especially in those younger than 2 years, a moderate number of positive NF and GFAP structure on the spot of the trauma was noted in comparison with the spot on the counterstroke where higher number of positive NF and GFAP structures was seen. That could be explained by a specific brain tissue development features in children. The data about the brain development stages has shown that in children up to 2 years of age the central nervous system (CNS) is actively developing and maturing [18]. If during this period of life CNS has some impact of mechanical force, necrotic and apoptotic death of CNS cells is more dynamic than in the children older than 2 years and adults [13]. This way with a high probability we can speculate that the dynamic IF forming mechanism in the white substance of the brain in children right after the trauma shows the plasticity and self-protection of the brain by forming a glial scar, and restricting the primary injury and limiting the spread of the secondary injury, including the degrading impact of inflammatory mediators on the penumbral tissue and the healthy brain tissue. Also, several manifestations of activated glia must be taken into account. While the glial response can provide trophic end metabolic support to the damaged neurons, deactivating toxins and eliminating cell debris, there is a growing evidence that the response may also be detrimental to neurons, as activated microglia and astrocytes can produce a variety of potentially neurotoxic molecules that are implicated in neuronal degeneration $[8 ; 16]$. It is possible that the survived patients, especially those who received therapy for a longer period of time parallel to dynamic IF production also inflammatory mediators and other neurotoxic substances were dynamically produced; they did not promote homeostasis in the 
brain tissue, and accelerated brain tissue degeneration. The neuroinflammation following acute brain trauma plays a prominent role in both the pathological and reconstructive response of the brain to injury [15].

The pathology of the traumatic brain injury is still extremely complex. For example, the glial activation in the injured brain certainly represents a disturbance of normal brain physiology or the predictor of pathological condition. It is still controversial as to whether reactive gliosis is harmful or beneficial to the acutely injured brain [9; 10]. For future ideal biomarkers will provide information on the pathobiology of traumatic brain injury and facilitate better monitoring of the progression of the secondary damage, the response to treatment and the prediction of outcome. But the initial characterization of biomarkers will be mainly based on the methods for measurement of such biomarkers in the blood serum. The development of reliable and accessible biomarkers is likely to change the way the clinical studies of head injury are conducted, resulting in more mechanism driven, optimally timed therapies [21].

\section{CONCLUSIONS}

1. Higher quantities of intermediated filaments such as glial fibrillary acidic protein (GFAP) and neurofilament (NF) are characteristic in patients who survived after the head trauma in comparison to those, who died on the spot of the accident.

2. Children under 2 years of age with severe head trauma have more dynamic glial cell reaction than other patients.

\section{REFERENCES}

1. Berger R.P. (2006) The use of serum biomarkers to predict outcome after traumatic brain injury in adults and children. J Head Trauma Rehabil, 21, 315-333.

2. Boloventa P., Wandosell F., Nieto-Sampedro M. (1992) CNS glial scar tissue: A source of molecules which inhibit central neurite outgrowth. Prog Brain Res, 94, 367-379.

3. Bonthius D.J., Steward O. (1993) Induction of cortical spreading depression with potassium chloride upregulates levels of Messenger 
RNA for glial fibrillary acidic protein in cortex and hippocampus: inhibition by MK-801. Brain Research, 618, 83-94.

4. Buffo A., Rolando C., Ceruti S. ( 2010) Astrocytes in the demaged brain: Molecular and cellular insights into their reactive response and healing potential. Biochem Pharm, 79, 77-89.

5. Chen Y., Swanson R.A. (2003) Astrocytes and Brain Injury. J Cereb Blood Flow Metab, 23, 137-149.

6. Frijns C.J.M., Kappelle L.J. (2002) Inflammatory cell adhesion molecules in ischemic cerebrovascular disease. Stroke, 33, 2115-2122.

7. Grände P.O. (2000) Pathophysiology of brain insult. Schweiz Med Wochenschr, 130, 1538-1543.

8. Hauwel M., Furon E., Canova C., Griffiths M., Neal J., Gasque P. (2005) Innate (inherent) control of brain infection, brain inflammation and brain repair: the role of microglia, astroytes, "protective" glial stem cells and stromal ependymal cells. Brain Reserch Rev, 48, 220-233.

9. John G.R., Lee S.C., Song X., Rivieccio M., Brosnan C.F. (2005) IL-1regulated responses in astrocytes: relevance to injury and recovery. Glia, 49,161-176.

10. Kimelberg H.K. (2005) Astrocytic swelling in cerebral ischemia as a possible cause of injury and target for therapy. Glia, 5, 389-397.

11. Kochanek P.M., Berger R.P., Bayir H., Wagner A.K., Jenkins L.W., Clark R.S. (2008) Biomarkers of primary and evolving damage in traumatic and ischemic brain injury: diagnosis, prognosis, probing mechanisms and therapeutic decision making. Curr Opin Crit Care, 14, $135-141$.

12. Laird M.D., Vanderm J.R., Dhandapani K.M. (2008) Opposing Roles for Reactive Astrocytes following Traumatic Brain Injury Neurosignals, $16,154-164$.

13. Lenroot R.K., Giedd J.N. ( 2006) Brain development in children and adolescents: Insights from anatomical magnetic resonance imaging. Neuroscience and Biobehavioral Reviews, 30, 718-729.

14. Liem R.K.H., Messing A. ( 2009) Dysfunctions of neuronal and glial intermediate filaments in disease. J of Clin Invest, 119(7), 1814-1824.

15. Lucas S.M., Rothwell N.J., Gibson R.M. (2006) The role of inflammation in CNS injury and disease. J Pharmacol, 147 (S1), S232-S240.

16. Mrak R., Griffin W.S. (2005) Glia and their cytokines in progression of neurodegeneration. Neorobiol Aging, 26, 349-354.

17. Norton W.T., Aquino D.A., Hozumi I., Chiu F.C., Brosnan C.F. (1992) Quantitative aspects of reactive gliosis: a review. Neurochem Research, $17,877-885$. 
18. Paus T., Collins D.L., Evans A.C., Leonarda G., Pikea B., Zijdenbos A. (2001) Maturation of white matter in the human brain: a review of magnetic resonance studies. Brain Research Bulletin, 54 (3), 255-266.

19. Pekny M., Nilsson M. (2005) Astrocyte activation and reactive gliosis. Glia, 50, 427-434.

20. Pilmane M., Luts A., Sundler F. (1995) Changes in Neuroendocrine Elements in Bronchial Mucosa in Chronic Lung Diseases in Adults. Thorax, 50, 551-554.

21. Pineda J.A., Wang K.K.W., Hayes R. (2004) Biomarkers of proteolytic demage following traumatic brain injury. Brain Pathology, 14, 202209.

22. Silver J., Miller J.H. (2004) Regeneration beyond the glial scar. Nat Rev Neurosci, 5, 146-156.

23. Stanimirovic D., Satoh K. (2000) Inflammatory mediators of cerebral endothelium: a role in ischemic brain inflammation. Brain Pathol, 10, $113-126$.

24. Streit W.J., Mrak R.E., Griffin W.S.T. (2004) Microglia and neuroinflammation: a pathological perspective. J of Neuroinflammation, 1, 14.

25. Williams A.J., Wei H.H., Dave J.R., Tortella F.C. (2007) Acute and delayed neuroinflammatory response following experimental penetrating ballistic brain injury in the rat. J of Neuroinflammation, 4, 17.

\section{Address for correspondence:}

Arta Barzdina

Riga Stradin's University

Department of Paediatric Intensive Care

Vienibas gatve 45, Riga, LV 1004;

E-mail: vetras17@inbox.lv 